\title{
Innovative immunotherapy targeting at PD-1/PD-L1 signaling pathway: mechanism, efficacy and safety analysis of monotherapy and combination therapies in non-small cell lung cancer (NSCLC) treatment
}

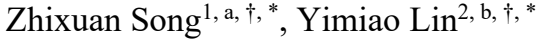 \\ ${ }^{1}$ Liaoning Province Shiyan High School, Shenyang, Liaoning Province 110010, China \\ ${ }^{2}$ School of International Education, Beijing University of Chemical Technology, Beijing 102200, P.R. China \\ †These authors contributed equally.
}

\begin{abstract}
Lung cancer remains a leading cause of cancer-related mortality worldwide with a poor prognosis. Conventional therapies are most commonly used in all kinds of treatment because of their relatively high efficacy in killing tumor cells at first. However, as treatment time increases, this efficacy would gradually decrease, along with unavoidable and growing resistance and multiple and serious side effects. At this point, immunotherapy, including anti-PD-1 and anti-PD-L1 antibodies, renders an innovative and more effective way to take advantage of our own immune response to kill cancer cells. It is confirmed to have greater efficacy and safety of immunotherapy over conventional therapies in various cancer treatments, including non-small cell cancer. Combining conventional therapies can also lead to synergistic effects in controlling and killing cancer cells. The purpose of this summary is to verify the efficacy and safety of immune checkpoint inhibitor monotherapy and the synergistic effects of combination therapy with chemotherapy and radiotherapy. This review will introduce the mechanism, efficacy, and safety of immune checkpoint inhibitor monotherapy and combination therapies with chemotherapy and radiotherapy via a summary and interpretation of related preclinical and clinical trials.
\end{abstract}

\section{Introduction}

Lung cancer, characterized by a wide range of tumor cell growth in the tissue of lung, includes more than 50 histomorphological subtypes, where non-small cell lung cancer (NSCLC) claims about 80\%-85\% confirmed cases [1]. There are 3 common types of NSCLC, adenocarcinomas (outer area of lung), squamous cell carcinomas (the center of the lung next to a bronchus), and large cell carcinomas (any part of the lung). NSCLC is one of the most common cancer types, known for its low recovery rate and high mortality rate because of late diagnosis [2]. Clinically, over half of lung cancer patients are diagnosed at stage III or IV (locally advanced or metastatic disease), at which the diseases grow out of control. Currently, most lung cancer treatments still rely on conventional chemotherapy and radiotherapy. Although these conventional therapies are effective in controlling and decreasing tumor size at first, they would also bring various kinds of serious adverse effects. Plus, these therapies will be less effective with the increase of treatment time.

Cancer cells can grow freely in that they can turn off our immune response and escape from immune surveillance conducted by $\mathrm{T}$ cells. Based on this mechanism of the escaping of cancer cells, immunotherapy was designed and investigated into and applied to cancer treatments over the past few decades. It reactivates our own immune response to fight against cancer cells, which lends it peculiarly high efficacy, safety and tolerability. One kind of immunotherapy agents is immune checkpoint inhibitor (ICI), including anti-PD-1 and anti-PD-L1 antibodies. It can block the PD-1/PD-L1 signaling pathway and reactivate $\mathrm{T}$ cells to proceed with immunological surveillance, thus effectively and safely clearing tumor cells. Currently, only two PD-1 antibodies (pembrolizumab and nivolumab) and three PD-L1 antibodies (atezolizumab, durvalumab, and avelumab) have been approved by the United States Food and Drug Administration (US-FDA) for first- or second-line cancer treatments [3]. Other agents are still being tested for efficacy and safety before they are approved for clinical use. In this review, we introduce the mechanisms of the PD-1/PD-L1 signaling pathway, its blockage by ICIs and acquired resistance to ICIs and summary both preclinical and clinical trials testing the efficacy, safety and tolerability of ICIs, pembrolizumab and atezolizumab in particular. We aim to confirm the improvement of immunotherapy in efficacy, safety, and tolerability compared with traditional chemotherapy and radiotherapy.

\footnotetext{
*Corresponding author: 2019090121@mail.buct.edu.cn. zhixuansong1022@163.com
} 


\section{Immune checkpoint}

Immune checkpoints are a set of proteins expressed on $\mathrm{T}$ cells and tumor cells, involved in the escape of cancer cells via interrupting the activation of T cells. Since they are the targets of immunotherapy, they form the basis of investigations into immunotherapy and appeal to many scientists' attention. Normally, activation of $\mathrm{T}$ cells in secondary lymphoid structures leads to the proliferation of effector $\mathrm{T}$ cells and onset of the immune response, which requires three distinct signals. Signal 1 is $T$ cell receptor (TCR) engagement of peptide-major histocompatibility complex (p-MHC) [8]. Signal 2 is costimulation between CD28 (cluster of differentiation 28) and B7-1 (cluster of differentiation 80 (CD80)) or B7-2 (cluster of differentiation 86 (CD86)), which controls the magnitude and duration of the response [4]. Signal 3 is inflammatory cytokines (such as IL-12 and type 1 interferon (IFN)) [5]. Accordingly, both antigen-specific and co-stimulatory signals are necessary for an optimal immune response. Immune checkpoints can deliver coinhibitory signals, which help maintain peripheral tolerance and prevents autoimmunity [6]. However, cancer cells can also be utilized to escape from the surveillance of T cells, which results in the uncontrollable growth of cancer cells. They include cytotoxic Tlymphocyte associated antigen-4(CTLA-4), PD-L1 (B7$\mathrm{H} 1$ or CD274) or PD-L2 (B7-DC or CD273) on tumor cells (TCs) and tumor-infilitrating immune cells (ICs), and PD-1(CD279) on T cells.

\subsection{CTLA-4 signaling pathway}

CTLA-4 interacts with PD-1 and PD-L1 (B7-H1 or CD274) or PD-L2 (B7-DC or CD273), which are the ligands of PD-1, by which it facilitates the escape of tumor cells from immune surveillance and lead to cancer progression and immune tolerance [7]. In the tumor microenvironment (TME), the shared ligands B7-1 (CD80) and B7-2 (CD86) on antigen-presenting cells deliver the costimulatory signal through CD28 and CTLA-4 on T cells. The T-cell response will be enhanced if signaling through CD28 and will be attenuated through CTLA-4 signalling [8]. CTLA-4 completely blocks costimulation by CD28 through its stronger affinity for B7 molecules [7]. The phenomenon of zipper-like oligomerization on the relatively small CTLA-4/B7-1 binding interface provides the structural basis to form unusually stable signaling complexes at the T-cell surface [8].

\subsection{PD-1/PD-L1 signaling pathway}

\subsubsection{Profile of PD-1/PD-L1}

Programmed cell death 1 (PD-1; also called CD279) and programmed death-ligand 1 (PD-L1; also called B7-H1 or CD274) are type I transmembrane proteins, and both of them belong to the immunoglobulin (Ig) superfamily. PD1 is an immune checkpoint, and it mainly consists of three important domains: one $\mathrm{lg}-\mathrm{V}$ like extracellular domain, one transmembrane domain, and one cytoplasmic domain with two tyrosine signaling motifs: ITIM (immunoreceptor tyrosine inhibitory motif) and ITSM (immuno receptor tyrosine-based switch motif). PD-L1 contains two extracellular domains (Ig-V- and Ig-C-like): a transmembrane domain and a short cytoplasmic tail which lacks known signaling motifs [7]. $\mathrm{T}$ cells in the tumor microenvironment (TME) highly express PD-1 after long exposure to tumor antigens. At the same time, tumor cells express high levels of PD-L1 when PD-L1 is expressed by tumour-infiltrating immune cells (ICs) [9].

The expression of PD-1 and PD-L1 is highly controlled by some transcription factors. The expression of PD-1 in antigen-activated $\mathrm{T}$ cells is modulated by nuclear factor of activated $\mathrm{T}$ cells, cytoplasmic 1 (NFATC1), fork-head box protein O1 (FOXO1), T-bet (also known as TBX21), B lymphocyte-induced maturation protein 1 (BLIMP1), and the serine-threonine kinase glycogen synthase kinase 3 (GSK3) [7,10-12]. PD1 levels will be decreased on responding $\mathrm{T}$ cells if the activating antigen is acutely cleared and will be increased and then sustained if the antigen is not cleared [7]. Besides, the expression of PD-L1 is upregulated on tumor cells when exposed to interferon- $\gamma$ and other cytokines released by local activated T cells, resulting in tumor cells' resistance to T-cell immunity, especially within the tumor microenvironment [13].

\subsubsection{Function of $P D-1 / P D-L 1$}

PD-1 and PD-L1 foster the immunosuppressive microenvironment, invalidate immunological surveillance, and help tumor cells escape from T cells via various pathways [14-15]. Different from CTLA-4, PD1/PD-L1 pathway expresses its inhibitory function in a more indirect way. For MEK - ERK-MAP kinase pathway, PD-1 attenuates it via inhibiting activation of PLC- $\gamma 1$ and Ras. By altering this way, PD-1 is likely to influence a plethora of downstream biochemical events. Also, PD-1 can inhibit T cell functions by increasing the expression of transcription factors such as basic leucine zipper transcriptional factor ATF-like (BATF), which can further repress the expression of effector genes $[7,16]$. Eventually, the interaction of PD-1 with PD-L1 (PD-1 ligations) suppresses the activated $\mathrm{T}$ cell proliferation, cytokine release, and cytolytic activity of PD-1-positive T cells in the tumor environment, which causes activated $\mathrm{T}$ cells to be exhausted and have a low antitumor function, thus leading tumor cells to escape from host immune attack [17].

Interaction of PD-1 with PD-L1 causes dampens of the TCR-mediated $\mathrm{T}$ cell activation and inhibition of the CD28-mediated co-stimulation. PD-1 on $\mathrm{T}$ cells will change its conformation, translocate to dynamic TCR microclusters, and accumulate at the signaling central supramolecular activation cluster(c-SMAC) after it bind its ligands (PD-L1 and PD-L2) on tumor cells. The cytoplasmic tail of PD-1 becomes phosphorylated under the action of Src family kinases. And it is these phosphorylated tyrosine motifs that serve as a docking site of the tyrosine phosphatase SHP-2 and SHP-1. Between 
SHP-2 and SHP-1, there's some scientific evidence to persuade that recruitment of SHP-2 in proximity to TCR attenuates key TCR proximal signaling events, such as Lck-mediated phosphorylation of ZAP70, and affects downstream signaling pathways, including those involving phosphoinositide 3-kinase (PI3K) - AKT, RAS, extracellular-signal-regulated kinase (ERK), VAV, and phospholipase $\mathrm{C} \gamma$ (PLC $\gamma$ ) [7]. PD-1 ligation mainly targets two pathways: PTEN-PI3K-Akt and RAS-MEKERK signaling [16-17]. PTEN is a serine - threonine phosphatase, and it is mediated by CK2. It can oppose the activation of PI3K and inhibit the signals delivered by the PI3K - Akt pathway. By recruiting SHP-2, PD-1 blocks the activation of PI3K. Although CK2 phosphorylates and stabilizes PTEN, it suppresses PTEN phosphatase activity during $\mathrm{T}$ cell activation. PD-1 causes diminished PTEN abundance but increased PTEN phosphatase activity by inhibiting the stabilizing phosphorylation of PTEN [7, 17].

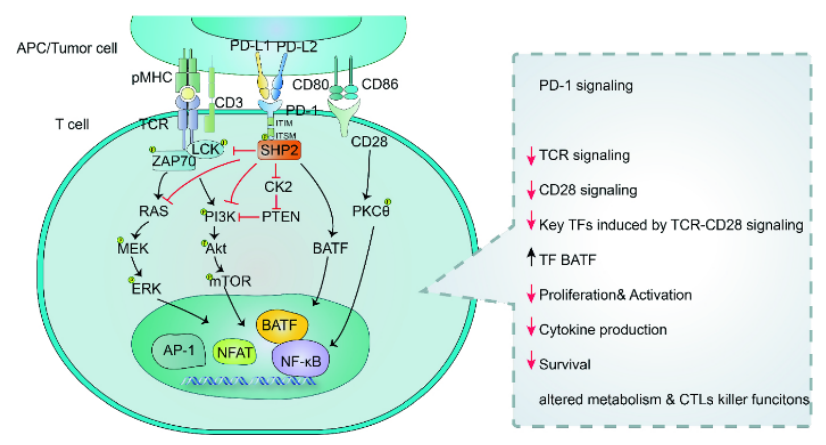

Fig. 1. PD-1/PD-L1 signaling pathway

\section{Immune checkpoint inhibitor}

\subsection{Profile}

As previously mentioned, the interaction of immune checkpoints and their ligands causes activated $\mathrm{T}$ cells to become exhausted and have a low antitumor function [6]. By interacting with the receptors and suspending the delivery of coinhibitory signals, ICIs blocking PD-1/PD$\mathrm{L} 1$ interaction can rescue these exhausted $\mathrm{T}$ cells and improve their anticancer functions [17]. In this way, active immunotherapy boosts the endogenous immune response via cancer vaccines or inhibitors of immune checkpoints.

Immune checkpoints involve CTLA-4, PD-1, and PDL1, etc. In accordance with their acting site, ICIs can be further divided into CTLA-4, PD-1 and PD-L1 inhibitors. PD-1/PD-L1 inhibitors block PD-ligand 1 or PD-ligand 2 from binding to $\mathrm{PD}-1$, therefore allowing $\mathrm{T}$ cell activation and immune system recognition $[19,20]$. This inhibition can be targeted at either PD-1 on T cells or PD-L1 on TCs or ICs. PD-1 inhibitors include nivolumab, pembrolizumab, tislelizumab, etc. Anti-PD-L1 inhibitors include atezolizumab, durvalumab, avelumab, etc. In this review, we mainly focus our attention on pembrolizumab and atezolizumab as respective representatives of antiPD-1 and anti-PD-L1 antibodies.

\subsection{Acquired resistance to immune checkpoint inhibitors}

Although the efficacy of immunotherapy is greater and more durable than that of conventional therapies, it is still ineluctable that patients will develop resistance to immunotherapy in the course of the treatments. Tumor cells can negatively regulate the expression of MHCantigen complexes to prevent recognition by effector $\mathrm{T}$ cells. Since the recognition of antigenic peptides is necessary for effective T cell-mediated response to ICIs, the loss of antigen and MHA molecules presentation leads to acquired resistance to ICIs [14]. Scientists have identified that immunoediting can trigger the development of tumor clones with low MHC (major histocompatibility complex) expression or poor bind between neoantigens and MHC during tumorigenesis $[14,21,22]$. PD-1 blockade can reinvigorate exhausted CD8 $+\mathrm{T}$ cells (Tex), restoring their anti-tumor effector functionality [23]. When CD8 $+\mathrm{T}$ cells are infiltrated in the TME, it can increase dysfunction driven by chromatin remodeling and epigenetic modifications and eventually reach a state of fixed epigenetic dysfunction in which their chromatin is rendered inaccessible and is thus resistant to further remodeling and reinvigoration by anti-PD-1 therapy. Therefore, a lack of memory $\mathrm{T}$ cells and reexhausted CD8 + T cells might lead to acquired resistance to PD-1 blockade [14,24,25].

Mutations within IFN signaling elements develop following PD-1/PD-L1 blockade, and they can prevent IFN- $\gamma$ signaling, PD-L1 expression, and acquired resistance to anti-PD-1 therapy. IFN- $\gamma$ leads to an upregulation of PD-L1 expression in tumors, increased antigen production, and increased release of $\mathrm{T}$ cellattracting chemokines [26]. Therefore, deletion of genes necessary for IFN- $\gamma$ signaling can lead to resistance to anti-PD-1/PD L1 therapies, playing a role in acquired resistance. Besides IFN- $\gamma$ signaling, expression of other immune checkpoint molecules, including CTLA-4, T cell immunoglobulin and mucin domain-containing molecule3 (TIM-3), lymphocyte activation gene-3 (LAG-3), and $\mathrm{V}$-domain Ig suppressor of T cell activation (VISTA), etc. has been proposed to lead to acquired resistance to PD1/PD-L1 blockade [14,27]. 


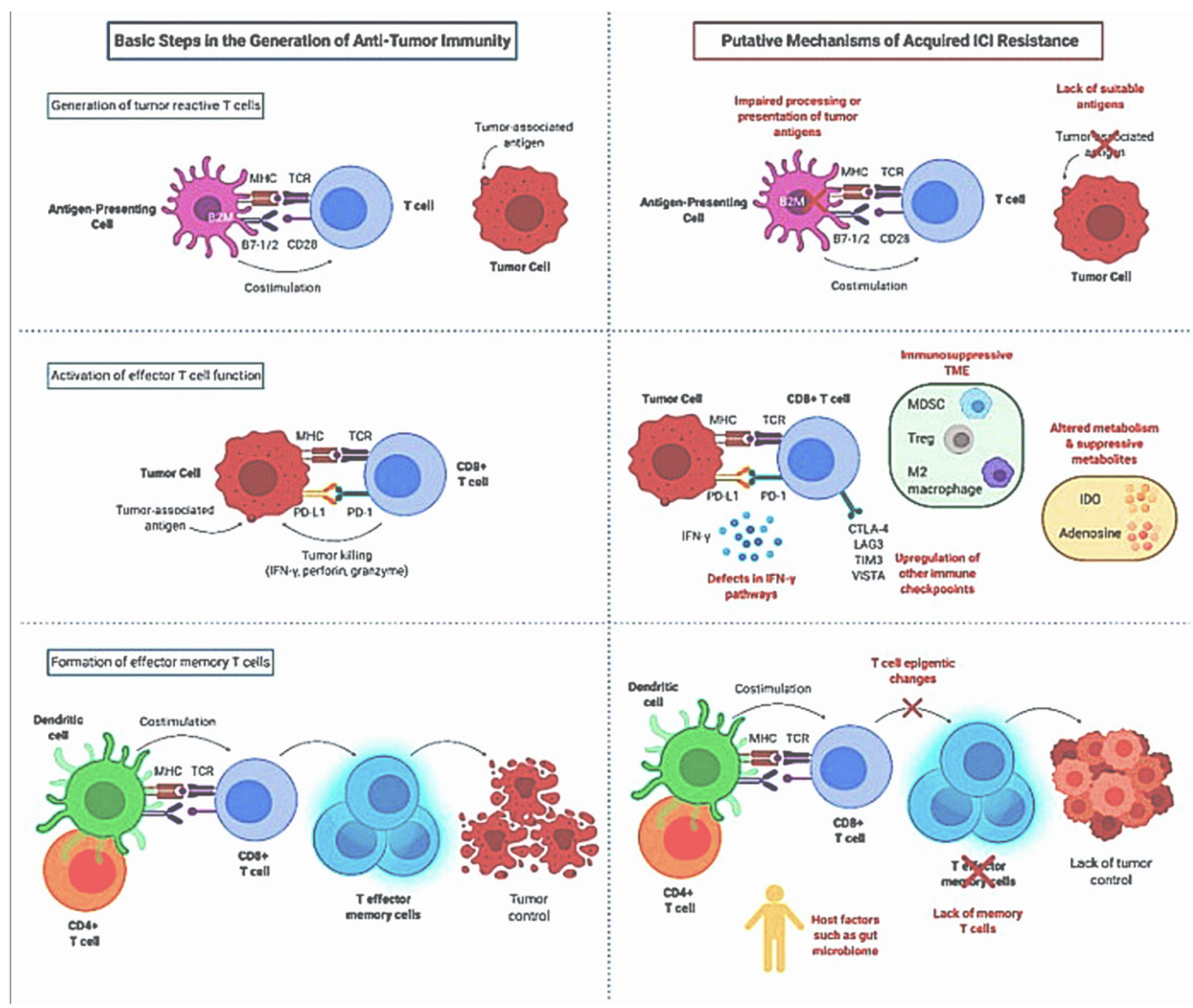

Fig. 2. Basic steps in the generation of anti-tumor immunity versus putative mechanisms of acquired ICI resistance

\section{Efficacy and safety}

While mechanisms need to be investigated into designing the ICI therapies, it is also important to test the efficacy and safety of ICIs in clinical trials before the official approvement of certain ICI usage in cancer treatment. Clinical trials show that ICIs can improve the overall survival, progression-free survival, and adverse events rate in patients with NSCLC, regardless of PD-L1 expression on tumor cells (TC) and tumor-infiltrating immune cells (IC) and previous treatment of the patients. The following section will focus on the evaluation of the efficacy and safety of pembrolizumab, a PD-1 inhibitor, and Atezolizumab, a PD-L1 inhibitor in clinical trials, which is compared with those of chemotherapy.

\subsection{Pembrolizumab versus chemotherapy}

Pembrolizumab is approved to treat a variety of cancer, including NSCLC, breast cancer, cervical cancer, among others, under FDA's Accelerated Approval Program, for its promising efficacy, safety and tolerability [3]. Pembrolizumab monotherapy greatly improves the overall survival, progression-free survival and incidence of adverse events of NSCLC patients in clinical trials.

KEYNOTE-024 Clinical Trials (NCT02142738) selected and randomly assigned 305 patients with untreated NSCLC (1:1) and TPS of $50 \%$ or greater to receive either pembrolizumab treatment or chemotherapy [28]. Compared with the chemotherapy group, the overall survival rate $(80.2 \% \mathrm{vs} 72.4 \%)$, response rate $(44.8 \% \mathrm{vs} 27.8 \%)$ and median progression-free survival (10.3months vs 6.0months) all increased considerably in the pembrolizumab group. Grade 3 or greater treatmentrelated adverse events (TPAEs) occurred in twice as many patients in the chemotherapy group as in the pembrolizumab group (53.3\% vs. 26.6\%). The occurrence of discontinuation of treatment because of TRAEs was decreased by $3.6 \%$ in the pembrolizumab group $(7.1 \% \mathrm{vs}$ $10.7 \%$ ). And there are three times as many cases of death related to treatment in the chemotherapy group (one death due to pulmonary sepsis on day 25 , one death due to pulmonary alveolar hemorrhage on day 112 , and one death of unknown cause on day 8) as those in 
pembrolizumab group (sudden death of unknown cause on day 2.

KEYNOTE-010 trial (NCT01905657) focused on patients with previously treated NSCLC. 1034 patients were randomly assigned them to receive $2 \mathrm{mg} / \mathrm{kg}$ pembrolizumab (345), $10 \mathrm{mg} / \mathrm{kg}$ pembrolizumab (346), or $75 \mathrm{mg} / \mathrm{m}^{2}$ docetaxel (343) every 3 weeks [29]. As a result, overall survival was significantly longer with pembrolizumab $2 \mathrm{mg} / \mathrm{kg}$ and $10 \mathrm{mg} / \mathrm{kg}$ than with docetaxel (median $14 \cdot 9$ vs 17.3 vs 8.2 months). Progression-free survival was also increased by pembrolizumab $2 \mathrm{mg} / \mathrm{kg}$ and $10 \mathrm{mg} / \mathrm{kg}$ compared with docetaxel (median 5.0 vs 5.2 vs 4.1 months). Pembrolizumab also improved 10-month overall survival rate and progression-freee survival rate compared with docetaxel (33.4\%vs35.8\%vs23.0\%,13.3\%vs $17.3 \% \mathrm{vs} 7.8 \%$ ).

Incidence of Grade 3 or greater adverse events attributed to study treatment was $43(13 \%)$ of 339 patients in the pembrolizumab $2 \mathrm{mg} / \mathrm{kg}$ group, 55 (16\%) of 343 patients in the pembrolizumab $10 \mathrm{mg} / \mathrm{kg}$ group, and $109(35 \%)$ of 309 patients in the docetaxel group. Incidence of discontinuation of treatment due to adverse events was $4 \%, 5 \%, 10 \%$, respectively. Cases of death related to treatment were 3,3 and 5, respectively. The overall survival and progression-free survival of patients in the pembrolizumab $10 \mathrm{mg} / \mathrm{kg}$ group were longer by $16.9 \%$ and $4 \%$ than $2 \mathrm{mg} / \mathrm{kg}$ group. This suggests pembrolizumab takes effects in a dose-dependent manner.

KEYNOTE-042 clinical trial (NCT02220894) included 1279 patients with previously untreated locally advanced or metastatic non-small-cell lung cancer, without a sensitizing EGFR mutation or ALK translocation and with tumor proportion score of $50 \%$ or greater, $20 \%$ or greater and $1 \%$ or greater [30]. They were randomly assigned to receive $200 \mathrm{mg}$ pemobrolizumab every 3 weeks for up to 35 cycles or platinum-based chemotherapy for four to six cycles. And patients, regardless of their TPS, all experienced longer median overall survival values than chemotherapy group, 20.0 months vs 12.2 months in TPS of $50 \%$ or greater group and chemotherapy group, 17.7 months vs 13.0 months in TPS of $20 \%$ or greater group and chemotherapy group, and 16.7 months vs 12.1 months in TPS of $1 \%$ or greater group and chemotherapy group. Median progression-free survival was also longer for the pembrolizumab group than the chemotherapy group, 7.1 months vs 6.4 months, 6.2 months vs 6.6 months, and 5.4 months vs 6.5 months,respectively. Incidence of TRAEs of grade 3 or greater was improved by $23 \%$ in the pembrolizumab group.

\subsection{Antezolizumab versus chemotherapy}

Comfirmatory trials also show that Atezolizumab monotherapy is well efficacious, safe, and tolerable in the treatment of NSCLC compared with historical chemotherapy. IMpower110(NCT02409342) enrolled 572 patients with previously untreated metastatic nonsquamous or squamous NSCLC and PD-L1 expression on at least $1 \%$ of tumor cells or at least $1 \%$ of
ICs and randomly assigned them to receive atezolizumab or chemotherapy [31]. The median overall survival was longer by 7.1 months in the atezolizumab group than in the chemotherapy group. 12-month overall survival rate was slightly improved in atezolizumab group $(57.6 \%$ vs $54.3 \%$ ). Incidence of all-grade adverse events and grade 3 or more adverse events was reduced by $4.5 \%$ and $22.4 \%$, respectively, in atezolizumab group.

In OAK(NCT02008227), 850 patients with previously treated NSCLC were randomly assigned (1:1) to receive atezolizumab monotherapy or docetaxel chemotherapy [32]. Patients in atezolizumab group benefited in longer overall survival (20.5 vs 8.9 months) and longer progression-free survival than cehmotherapy group (7.8 vs 3.9 months). Overall survival was increased by 3.7 months in patients with low expression of PD-L1 and 11.6 months in patients with high expression of PD-L1. 12month overall survival rate was also improved in atezolizumab group compared with the chemotherapy group $(55 \%$ vs $41 \%)$. In patients with non-squamous NSCLC , 12-month progression-free survival was increased by $16.9 \%(55.9 \%$ vs $39 \%)$. And in patients with squamous NSCLC, it was increased by $13 \%$ (38.4\% vs $25.4 \%$ ). Incidence of grade 3 or greater adverse events, discontinuation of treatment, and case of death related to adverse events were decreased by $17 \%, 11 \%$ and $0.2 \%$ in pembrolizumab group.

POPLAR (NCT01903993) selected 287 patients with previously treated squamous and non-squamous NSCLC to receive either atezolizumab or docetaxel treatment [33]. The overall survival of patients with squamous NSCLC was improved by 1.5 months in atezolizumab group (10.1 vs 8.6 months). For the non-squamous patients, overall survival was also prolonged by 4.6 months in atezolizumab group (15 5 vs 10.9 months). 12-month overall survival rate was improved by $10.1 \%$ in atezolizumab group (47.9\% vs $37.8 \%$ ). Grade-3 or more adverse events are less frequent in atezolizumab group than docetaxel group (15\% vs 43\%). Patients with PD-L1 expressing on $50 \%$ or greater of tumor cells (TC3) or tumor infiltrating immune cells(IC3) derived the greatest benefits from antezolizumab monoptherapy as their progression-free survival was doubled(7.8 vs 3.9 months).

BIRCH(NCT02031458) selected 659 patients with TPS of 5\% or greater, which comprised 3 cohorts, cohort 1 (no prior chemotherapy), cohort 2 (one prior platinum chemotherapy) and cohort 3 (at least two chemotherapies) [34]. The median duration of survival follow-up for all treated patients was 14.6 months, with 20.1 months in cohort1, 15.5 months in cohort 2 and 13.2 months in cohort 3 . And the median progression-free survival was 5.2 months, 2.8 months, 2.8 months, respectively. 12month overall survival rate and progression-free survival were $66.4 \%, 58.1 \%$ and $52.3 \% ; 20 \%, 17 \%$, and $14 \%$ for cohort1, 2, and 3, respectively. Incidence of grade 3 or greater adverse events was $42 \%$ and discontinuation of treatment was $7 \%$ in 3 cohorts. However, no clear relationship between previous treatment and efficacy of atezolizumab could be drawn from BIRCH results.

FIR phase II study (NCT01846416) enrolled 3 cohorts (patients without platinum-based chemotherapy for 
metastatic disease or adjuvant therapy within 6 months of recurrence in cohort 1; patients with prior platinum-based chemotherapy for metastatic disease and without brain metastases in cohort 2; patients with prior platinum-based chemotherapy for metastatic disease and brain metastases in cohort 3) and assigned them to receive $1200 \mathrm{mg}$ of intravenous atezolizumab on the first day of 21-day cycles [35]. Median Progression free survival was 5.5 months for Cohort 1, 3.7 months for Cohort 2, and 4.3 months for Cohort 3. Incidence of all grade TRAES was $70 \%$ in 3 cohorts: $81 \%$ in Cohort $1,67 \%$ in Cohort 2 , and $69 \%$ in Cohort 3. 12-month overall survival rate and progressionfree survival were $25.8 \%, 19.3 \%$ and not reached; $67.7 \%$, $38.7 \%$ and $38.5 \%$ for cohort $1,2,3$. Incidence of grade 3 or greater TRAEs were $18 \%$ altogether: $16 \%, 19 \%$, and $15 \%$, respectively. Still, there was no definite relation between prior treatment and the efficacy of antezolizumab.

\subsection{PD-L1 expression on TC and IC as predictive biomarkers}

From the results of clinical trials, there is a clear positive relation between PD-L1 expression level and the efficacy of pembrolizumab: the overall survival and progressionfree survival improve as the expression level increases, which is consistent with the mechanism of pembrolizumab.

PD-L1 expression on TCs can serve as predictive biomarkers for the extent of the response to immunotherapy in the treatment of NSCLC. In KEYNOTE-001(NCT01295827), overall survival and progression free survival of patients with PD-L1 expression on $50 \%$ or more TC or IC were all the longest among all the patients with $50 \%$ or greater, $1 \%-49 \%$, less than $1 \%$ TC or IC [36]. 6-month and 12-month overall survival rate were also the largest in $50 \%$ or greater TC or IC: $50.4 \%$ and $10.9 \%$ in $50 \%$ or greater; $27.9 \%$ and $0.6 \%$ in $1 \%-49 \% ; 22.3 \%$ and $2.6 \%$ in less than $1 \%$. KEYNOTE-042 also showed that median overall survival was increased by 7.8 months in patients with TPS of $50 \%$ or greater, 4.7 months in TPS of $20 \%$ or greater, and 4.6 months in patients with TPS of $1 \%$ or greater [30].

Likewise, higher PD-L1 expression on ICs also contributes to greater efficacy of immunotherapy in treatment of NSCLC. In POPLAR, the greatest improvement of overall survival among all the patients enrolled was 3.9 months in patients with PD-L1 expression on $50 \%$ or greater TC or IC [35]. POPLAR further analyzed the respective influence of PD-L1 expression on TC and IC on efficacy of antezolizumab. For patients with PD-L1 expression on tumor cells only, median overall survival was increased by 1.2 months with atezolizumab compared with docetaxel (13.2 vs 12 months). And for patients with PD-L1 expression on ICs only, median overall survival was increased by 4.5 months with atezolizumab compared with docetaxelmedian(14.3 vs 9.8 months).

\subsection{Different types of adverse events related to immunotherapy and chemotherapy}

Compared with chemotherapy, immunotherapy also unavoidably brings about adverse events, but to a relatively more moderate extent. Common adverse events in the treatment of NSCLC include fatigue, pruritus, decreased appetite, rash arthralgia, diarrhea, nausea, hypothyroidism, asthenia, anemia, dyspnea, pyrexia, and so on. [30-36] ICIs will be more likely to trigger off immune-mediated adverse events, which confirms the mechanism of the effects of ICIs. Patients treated by ICIs are actually more susceptible to Endocrine irAEs, including hypophysitis, thyroid dysfunctions, Type 1 diabetes mellitus (T1DM) and so on [37]. In KEYNOTE024 [28], although Grade 3 or more TRAEs rate in the pembrolizumab group was approximately reduced to half of that in the chemotherapy group (26.6\% vs. $53.3 \%)$, immune-mediated adverse events of all kinds actually occurred more frequently in pembrolizumab group than chemotherapy group (29.2\% vs $4.7 \%)$. Another case in point is the comparison of the results in KEYNOTE042[3], in which the most common adverse events also differed between the pembrolizumab and chemotherapy group: hypothyroidism (69 [11\%] of 636) in the pembrolizumab group and anaemia (229 [37\%] of 615) in chemotherapy group. The divergence in the incidence of hypothyroidism can probably be attributed to the expression of PD-L1 and PD-L2 on thyroid gland cells $[38,39]$. Nevertheless, since the grade of immune-related adverse events was mostly moderate and was thus less common to induce death, the overall safety profile was still better with pembrolizumab group than with chemotherapy group.

\section{Combination therapy}

ICIs can significantly improve the overall survival, progression-free survival, and occurrence of adverse events in NSCLC patients. However, many patients have primary resistance to immunotherapy. Only $20 \%-50 \%$ of them in previous trials can benefit from immunotherapy $[40,41]$. It is also unavoidable that patients develop acquired resistance to immunotherapy in the course of treament. Moreover, the efficacy and safety of ICIs are highly dependent on PD-L1 expression on both TCs and ICs. However, what is still unclear to scientists is the definite minimum score of the PD-L1 expression, which the patients must have to benefit from immunotherapy. Given the respective advantages and disadvantages of immunotherapy and conventional therapies, scientists are considering combining the two of them to realize synergistic effects in cancer treatments, which are to be confirmed in the following section.

\subsection{Combination with chemotherapy}

Chemotherapy, particularly platinum-based doublet chemotherapy, constitutes the basis of the first-line treatment of advanced NSCLC. Since most chemotherapeutic agents are immunosuppressants, it has 
long been deemed as incompatible with combining the two therapies together [42]. Actually, the combination of chemotherapy and immunotherapy can enhance the efficacy of immunotherapy, which is even greater than that of historical immunotherapy. But clinical trials didn't observe any significant improvement in the safety profile of the immunotherapy plus chemotherapy group. The reason for this synergistic effect is that chemotherapy can induce immunogenic tumor cell death; reduce the number of immunosuppressive cells, including regulatory $\mathrm{T}$ cells and Myeloid derived suppressor cell(MDSCs) [43,44]; promote the maturation of antigen-presenting cells, including dendritic cells [45]; upregulate the expression of MHC I on tumor cells to sensitive tumor cells to immune cells [46], etc.

KEYNOTE-189(NCT02578680) selected 616 Patients with previously untreated pathologically confirmed metastatic nonsquamous NSCLC without sensitizing EGFR/ALK alterations [47]. They were randomly assigned $(2: 1)$ to receive pemetrexed and platinum(chemotherapy) plus pembrolizumab or placebo every 3 weeks for 4 cycles, then pemetrexed maintenance plus pembrolizumab or placebo for up to a total of 35 cycles. Overall survival and progression-free survival were longer in the combination therapy group than the chemotherapy group (22.0 vs 10.7 months, 9.0 vs 4.9 months). 12-month overall survival rate and progressionfree survival rate were almost doubled in the pembrolizumab plus chemotherapy group ( $70 \%$ vs $48.1 \%$, $38.8 \%$ vs $16.8 \%$ ), which was also greater than those in the pembrolizumab alone group in KEYNOTE024(25.3\%,14.2\%) and KEYNOTE-042(23.8\%, 52.1\%). Yet, no significant difference was observed between the frequency of grade 3 or more adverse events between the two groups $(71.9 \%$ vs $66.8 \%)$ [30,32].

KEYNOTE-407(NCT02775435) enrolled patients with previously untreated pathologically confirmed metastatic nonsquamous NSCLC and without sensitizing EGFR/ALK alterations and then randomly assigned (1:1) them to receive carboplatin and paclitaxel/nabpaclitaxel(chemotherapy) plus either pembrolizumab 200 mg or saline placebo every 3 weeks [48]. Median overall survival was increased by 5.5 months (17.1 vs 11.6 months), and median progression-free survival was increased by 2.9 months ( 8.0 vs 5.1 months) in pembrolizumab plus chemotherapy group. 12-month overall survival rate and progression-free survival rate were increased by $15.1 \%(64.7 \% \mathrm{vs} 49.6 \%)$ and by $18.1 \%$ (35.8\% vs $17.7 \%$ ) in pembrolizumab plus chemotherapy group. Incidence of grade 3 or greater adverse events was similar between pembrolizumab plus chemotherapy group and pembrolizumab plus placebo group (56.5\%vs55.7\%).

In IMpower150(NCT02366143), patients with chemotherapy-naive metastatic NSCLC were randomly assigned $(1: 1: 1)$ to receive atezolizumab plus bevacizumab, carboplatin and paclitaxel (ABCP); atezolizumab plus carboplatin and paclitaxel (ACP); the bevacizumab, carboplatin and paclitaxel (BCP) [49]. Median overall survival was increased by 3.9 months in ABCP group (13.3 vs 9.4 months) and 2.7 months in ACP group (21.4 vs 18.7 months) compared with BCP group.
Similarly, no significant difference in safety profile was observed among the three groups, with grade 3 or more adverse events occurring in $57 \%, 43 \%$ and $49 \%$ patients, respectively.

IMpower130(NCT02367781) enrolled 724 patients with histologically or cytologically confirmed and previously untreated stage nonsquamous NSCLC and randomly assigned $(2: 1)$ them to receive $1200 \mathrm{mg}$ atezolizumab(every3weeks) plus $6 \mathrm{mg} / \mathrm{mL}$ per min carboplatin (every 3 weeks) and $100 \mathrm{mg} / \mathrm{m} 2 \mathrm{nab}$ paclitaxel (every week) or chemotherapy alone according to the same schedule for 4 or 6 cycles of 21 days [50]. Anteozlizumab combined with chemotherpay significantly improved the median overall survival by 4.7 months (18.6 vs 13.9 months) and median progressionfree survival by 1.5 months ( 7.0 vs 5.5 months). 12months overall survival rate and progression-free survival were increased by $15 \%(29.1 \%$ vs $14.1 \%)$ and $7.6 \%(63.1 \%$ vs $55.5 \%$ ). Incidence of serious adverse events was increased by $13 \%$ in atezolizumab plus chemotherapy group (51\% vs 38\%) and proportion of discontinuation of treatment was also increased by $4 \%(26 \%$ vs $22 \%)$. Cases of treatment-related death were 8 in the combination therapy group and 1 in the chemotherapy group. Immunerelated adverse events occurred in nearly half $(45 \%)$ of the patients in combination therapy group, but the majority of them were only grade1-2.

\subsection{Combination with radiotherapy}

Radiotherapy, currently used in local lesion eradication, includes stereotactic body radiation therapy (SBRT) or stereotactic ablative radiotherapy (SAR). Coventionally fractionated radiation can be quite effective in diminishing the tumor size at one time. Yet it is confronted with radioresistance both in vivo and in vitro, which is thought to be directly associated with increased expression of PD-L1 [51]. Similar to immunotherapy combined with chemotherapy, immunotherapy combined with radiotherapy can also result in better efficacy profile. This is probably because Radiotherapy can alleviate the immunosuppressive microenvironment and upregulate PD-L1 expression. In the mice model, compared with anti-PD-L1 alone, anti-PD-L1 combined with radiotherapy greatly increased $\mathrm{CD} 8+$ cells and reduced regulatory $\mathrm{T}$ cells and MDSCs [44]. It can also induce immunogenic cell death (ICD) in various ways [52]. In turn, immunotherapy can block PD-L1 on tumor cells and mediate radioresistance. The expression of PD-L1 was greatly reduced in anti-PD-L1 antibody plus radian group compared with anti-PD-L1 antibody alone, while that was increased in the radiotherapy group [53].

Preclinical trials have revealed synergistic effects of immunotherapy plus radiotherapy. G.S. Herter-Sprie and S. Koyama [53] observed that the percentage of overall survival and progression-free survival were all the greatest in the radian plus anti-PD-1 antibody group. And the tumor volume in the combination group remained relatively stable in a range of $65 \%-85 \%$ of initial volume while the tumor volume of both radiotherapy alone group and immunotherapy alone group increased considerably. 
Gong $\mathrm{X}$ and her colleagues [51] also found that combination of conventionally fractionated radiotherapy and anti-PD-L1 antibody can result in synergistic antitumor effects in the treatment of NSCLC in a mouse model. Tumor volume didn't expand but slightly decreased only in the anti-PD-L1 antibody plus the radian group while the only group of tumors in the anti-PD-L1 only group and the radian only group expanded at least 2.5-fold.

Clinical trials further suggest that previous treatment with radiotherapy can improve the overall survival and progression-free survival of NSCLC patients. PEMBRORT(NCT02492568) selected 78 patients with advanced NSCLC and randomly assigned $(1: 1)$ them to receive 200 mg pembrolizumab every 3 weeks either after SBRT (experimental group) or without SBRT (control group) [54]. The progression-free survival and overall survival were increased by 4.7 months ( 1.9 vs 6.6 months) and 8.3 months (7.6 vs 15.9 months) in the experimental group. 12-month progression-free survival rate and overall survival rate were nearly doubled in experimental group ( $15 \%$ vs $33 \%, 22.5 \%$ vs $50 \%$, respectively). There was no significant difference between the incidence of all grade and grade3-5 TRAEs of the experimental group and that of the control group. Yet, $18 \%$ in the experimental group (26\% vs $8 \%$ ) increased the incidence of pneumonitis.

A secondary analysis of KEYNOTE-001 compared the results of patients who received prior radiotherapy, including extracranial radiotherapy and thoracic radiotherapy, and those who didn't receive [55]. Median progression-free survival was increased by 2.3 months (4.4 vs 2.1 months) and median overall survival was increased by 5.4 months ( 10.7 vs 5.3 months) in patients who received any radiotherapy before compared with those who didn't. Likewise, median progression-free survival was increased by 4.3 months ( 6.3 vs 2.0 months) and median overall survival was increased by 6.3 months (11.6 vs 5.3 months) in patients who received extracranial radiotherapy before compared with those who didn't. But patients who received thoracic radiotherapy before were more susceptible to pulmonary toxicities. Incidence of allgrade treatment-related pulmonary toxicities of patients who received prior thoracic radiotherapy was $12 \%$ higher than that of patients who didn't $(13 \%$ vs $1 \%)$. And the incidence of grade 3 or greater treatment-related pulmonary toxicities was also $3 \%$ higher (4\% vs $1 \%$ ).

Based on previous clinical trials, ICIs treatment didn't improve the safety profile of patients with prior radiation treatments. In fact, ICIs were observed to increase the incidence of radiation recall pneumonitis(RRP). It is a unique pattern of radio-related toxicities induced by ICIs. It happens when ICIs evoke an inflammatory reaction in previously irradiated tumor sites, which is thought to be mediated by a series of cytokines (IL-4, 6, 10, 13, 17, 18) and relative signaling pathways $[58,59,60,61]$ RRP induced by immunotherapy has several idiosyncrasies: Firstly, it takes quite a long time for immunotherpayrelated RRP to surface after radiotherapy treatment. The interval between prior radiotherapy and diagnosis of RRP can be up to 2 years [60]. Secondly, immunotherapyrelated RRP might be associated with PD-L1 expression. Both Shibaki R. and Manapov F. observed that cases of immunotherapy-induced RRP all showed durable responses to immunotherapy $[60,61]$. From what has been discussed in 3.1, the efficacy of immunotherapy is highly dependent on the PD-L1 expression level. Therefore, there might be a positive relationship between PD-L1 expression and immunotherapy-induced RRP, which conforms to its hypothesized mechanism that PD-1 blockade can free PD-L2 from PD-1 ligation increase its chance of binding to repulsive guidance molecule $b$ (RGMb), leading to RRP. However, due to limited researches and data, we can still not tell a definitive relationship between the level of PD-L1 expression and the occurrence of immunotherapy-induced RRP [62].

\subsection{Combination with chemoradiotherapy}

Additionally, clinical trials revealed synergy of combining immunotherapy, chemotherapy and radiotherapy together. Phase 1 trial of pembrolizumab administered concurrently with chemoradiotherapy for locally advanced NSCLC(NCT02621398) [63] randomly assigned 6 patients to receive $100 \mathrm{mg}$ or $200 \mathrm{mg}$ pembrolizumab every 3 weeks after day 1 or 29 of chemoradiotherapy treatment. The median progressionfree survival was 18.7 months and the median overall survival was 29.4 months. And the 12-month progressionfree survival and overall survival rate were $69.7 \%$ and $85.2 \%$, which was quite promising compared with $25.3 \%$ and $14.2 \%$ in pembrolizumab alone group in KEYNOTE024 and $23.8 \%$ and $52.1 \%$ in KEYNOTE-042 [28, 30].

\section{Conclusion}

In this review, we systematically summarize the mechanism of immune checkpoint inhibitors in immunotherapy, pembrolizumab and atezolizumab in particular, and includes both preclinical and clinical trials to confirm the advantageous efficacy and safety profile in immunotherapy over conventional cancer treatments. Conventional cancer treatments, including chemotherapy and radiotherapy, are confronted with myriad problems, including relative low efficacy, high incidence of adverse events and primary or acquired resistance. Immunotherapy, on the other hand, has significantly improved performance in efficacy, safety, and tolerance. As we have discussed, ICIs, targeting the PD-1/PD-L1 signaling pathway, can reactivate immune surveillance to kill tumor cells, thus rendering immunotherapy considerable specificity and efficacy. Above-mentioned clinical trials have revealed a strong positive relation between the efficacy of ICIs and PD-L1 expression on both TCs and ICs: the higher the PD-L1 expression is, the longer the overall survival and progression-free survival are, the higher the overall survival rate and progressionfree survival rate are. In other words, PD-L1 expression on TC and IC may serve as predictive biomarkers to determine whether the patients can benefit from immunotherapy or not and further determine whether to apply it or not. What's more, this high profile of efficacy and safety can be further improved if immunotherapy is combined with conventional therapies, chemotherapy, 
and immunotherapy. According to what we have discussed, they can change the immunosuppressive microenvironment, which sheds light on combination therapies in NSCLC treatment.

The last few decades witnessed great advances in and deep insights into immunotherapy and ICIs, owing to increasing researches and clinical trials. Up till now, 6 ICIs, 5 PD-1/PD-L1 inhibitors, and 1 CTL4 inhibitor, have been approved by US-FDA for first or second-line cancer treatments. Immunotherapy is catching increasingly more attention from the scientific field for its high efficacy and safety, confirmed in previous clinical trials. However, there are still many challenges in the immunotherapy treatment of NSCLC. First, it is still unclear what definite minimum score of the PD-L1 expression the patients must have to benefit from immunotherapy. According to updated clinical data, only $20 \%-50 \%$ of patients with various kinds of cancer can really benefit from ICIs treatment. Patients with relatively high PD-L1 expression are tested to have longer overall survival and progression-free survival with ICIs treatment. And it is needed to be formally tested whether patients with lower PD-L1 expression can benefit from immunotherapy as well. Second, although ICIs therapies were more tolerable than conventional therapies, the responses can only last for up to 1 year. It is still inevitable for patients to have primary resistance or develop acquired resistance to immunotherapy. Third, the mechanisms behind the synergistic effects of combination therapies are largely unknown. For example, the mechanism that prior radiotherapy treatment can lead to better efficacy of following immunotherapy treatment and that immunotherapy induces RRP in the previously irradiated tumor site. The relationship between PD-L1 expression and immunotherapy-induced RRP is yet to be confirmed due to a lack of data. Hence, future researches should be oriented at defining the minimum score of PDL1 expression required for receiving immunotherapy and figuring out the underlying mechanisms behind primary and acquired resistance and combination therapies. With more available researches and data, combination therapies can be better designed to maximize the efficacy and safety and overcome acquired resistance to immunotherapy.

\section{References}

1. Osmani, Lais et al. Seminars in cancer biology vol. 52,Pt 1 (2018)

2. Siegel, Rebecca L et al. CA: a cancer journal for clinicians vol. 70,1 (2020)

3. Khanal, S. , \& Zhang, H. Journal of Biosciences and Medicines, 07(6): 1-12 (2019)

4. Bretscher P. A. Proceedings of the National Academy of Sciences of the United States of America vol. 96(1): 185 - 190 (1999)

5. Wei, S.C.; Duffffy, C.R.; Allison, J.P. Cancer Discovery vol. 8(9):1069 - 1086 (2018).

6. Boussiotis VA. The New England journal of medicine vol. 375(18):1767-1778 (2016)
7. Ai L, Xu A, Xu J. Adv Exp Med Biol. 1248:33-59 (2020)

8. Stamper CC, Zhang Y, Tobin JF, Erbe DV, Ikemizu S, Davis SJ et al. Nature vol. 410(6828):608-611 (2001)

9. Herbst RS, Soria JC, Kowanetz M, et al. Nature vol. 515(7528):563-567(2014)

10. Keir ME, Butte MJ, Freeman GJ, Sharpe AH. Annual review of immunology vol. 26:677-704(2008)

11. Schildberg FA, Klein SR, Freeman GJ, Sharpe AH. Immunity vol. 44(5):955 - 972(2016)

12. Taylor A, Harker JA, Chanthong K, Stevenson PG, Zuniga EI, Rudd CE. Immunity vol. 44(2):274 286(2016)

13. Sun C, Mezzadra R, Schumacher T.N. Immunity vol. 48(3):434 - 452.(2018)

14. Pathak R, Pharaon RR, Mohanty A, Villaflor VM, Salgia R, Massarelli E. Cancers vol. 12(12):3851(2020)

15. Iwai $\mathrm{Y}$, Ishida $\mathrm{M}$, Tanaka $\mathrm{Y}$, Okazaki $\mathrm{T}$, Honjo $\mathrm{T} \&$ Minato N. Proceedings of the National Academy of Sciences of the United States of America vol. 99(19), 12293-12297(2002)

16. Patsoukis N, Brown J, Petkova V, Liu F, Li L, Boussiotis VA. Science Signaling vol. 5(230):46(2012)

17. Patsoukis N, Li L, Sari D, Petkova V, Boussiotis VA. Molecular and cellular biology vol. 33(16):3091 3098 (2013)

18. Parry RV, Chemnitz JM, Frauwirth KA, Lanfranco AR, Braunstein I, Kobayashi SV et al. Molecular and cellular biology vol. 25:9543 - 9553(2005)

19. Paz-Ares L, Vicente D, Tafreshi A, et al.. Journal of thoracic oncology: official publication of the International Association for the Study of Lung Cancer vol. 15(10):1657-1669(2020)

20. Ellis PM, Vella ET, Ung YC. Clinical Lung Cancer vol. 18(5):444-459 (2017)

21. Anagnostou V, Smith KN, Forde PM, et al. Cancer Discovery vol. 7(3):264-276(2017)

22. Gettinger S; Choi J; Hastings K; Truini A; Datar, I.; Sowell R; Wurtz A; Dong W; Cai G; Melnick M.A. et al. Cancer Discovery vol.7(12):1420-1435(2017)

23. Wherry E.J, Kurachi M. Nature reviews. Immunology vol. 15(8):486 - 499(2015)

24. Philip M, Fairchild L, Sun L, Horste E.L, Camara S, Shakiba M, Scott A.C, Viale A, Lauer P, Merghoub T, et al. Nature vol. 545(7655): 452 - 456(2017)

25. Pauken K.E, Sammons M.A, Odorizzi P.M, Manne S, Godec J, Khan, O, Drake A.M, Chen Z, Sen D.R, Kurachi M, et al. Science( New York, N.Y.) vol. 354(6316): 1160 - 1165(2016)

26. Abiko K, Matsumura N, Hamanishi J, et al. British journal of cancer vol. 112(9):1501 - 1509(2015) 
27. Shin, D.S, Ribas, A. Current opinion in immunology vol. 33:23 - 35(2015)

28. Reck M, Rodríguez-Abreu D, Robinson AG, et al. The New England journal of medicine vol. 375(19):1823-1833(2016)

29. Herbst RS, Baas P, Kim DW, et al. Lancet (London, England) vol. 387(10027):1540-1550(2016)

30. Mok TSK, Wu YL, Kudaba I, et al. Lancet (London, England) vol. 393(10183):1819-1830(2019)

31. Herbst, Roy $\mathrm{S}$ et al. The New England journal of medicine vol. 383(14): 1328-1339(2020)

32. Rittmeyer, Achim et al. Lancet (London, England) vol. 389(10066):255-265(2017)

33. Fehrenbacher, Louis et al. Lancet (London, England) vol. 387(10030):1837-46(2016)

34. Peters, Solange et al. Journal of clinical oncology: official journal of the American Society of Clinical Ocology vol. 35(24):2781-2789(2017)

35. Spigel, David R et al. Journal of thoracic oncology: official publication of the International Association for the Study of Lung Cancer vol. 13(11):17331742(2018)

36. Garon, Edward B et al. The New England of medicine vol. 372(21):2018-2028(2015)

37. Mok TSK, Wu YL, Kudaba I, Kowalski DM, Cho BC, Turna HZ, Castro G Jr, Srimuninnimit V, Laktionov KK, Bondarenko I, Kubota K, Lubiniecki GM, Zhang J, Kush D, Lopes G. Lancet. 393(10183):18191830(2019)

38. Chowdhury S, Veyhl J, Jessa F, Polyakova O, Alenzi A, MacMillan C, Ralhan R, Walfish PG. Oncotarget vol. 7(22):32318-32328(2016)

39. Rosenbaum MW, Gigliotti BJ, Pai SI, Parangi S, Wachtel H, Mino-Kenudson M, Gunda V, Faquin WC. Endocrine Pathology vol. 29(1):59-67(2018)

40. Brahmer, Julie R at al. The New England journal of medicine vol. 366(26):2455-2465(2012)

41. Topalian, Suzanne L et al. The New England journal of medicine vol.366(26):2443-2454(2012)

42. Apetoh L, Ladoire S, Coukos G, Ghiringhelli F. Annals of oncology: official journal of the European Society for Medical Oncology vol.26(9):18131823(2015)

43. Apetoh L, Végran F, Ladoire S, Ghiringhelli F. Current molecular medicine vol. 11(5):365-72(2011)

44. Ghiringhelli F, Apetoh L. Biomedical journal vol. 38(2):111-6(2015)

45. Liu WM, Fowler DW, Smith P, Dalgleish AG. British journal of cancer vol. 102(1):115-23(2010)

46. Ramakrishnan R, Assudani D, Nagaraj S, Hunter T, Cho HI, Antonia S, Altiok S, Celis E, Gabrilovich DI. The Journal of clinical investigation vol. 120(4):1111-24(2010)

47. Gadgeel, Shirish et al. Journal of clinical oncology: official journal of the Americak Society of Clinical Oncology vol. 38(14):1505-1517(2020)
48. Paz-Ares, Luis et al. Journal of thoracic oncology: official publication of the International Association for the Study of Lung Cancer vol. 15(10):16571669(2020)

49. Reck, Martin et al. The Lancet. Respiratory medicine vol. 7(5):387-401(2019)

50. West, Howard et al. The Lancet. Oncology vol. 20(7):924-937(2019)

51. Gong X, Li X, Jiang T, Xie H, Zhu Z, Zhou F, Zhou C. Journal of thoracic oncology: official publication of the International Association for the Study of Lung Cancer vol. 12(7):1085-1097(2017)

52. Rapoport BL, Anderson R. International journal of molecular sciences vol. 20(4):959(2019)

53. Herter-Sprie Grit $\mathrm{S}$ et al. JCI Insight Vol. 1(9):e87415(2016)

54. Theelen, Willemijn S M E et al. JAMA oncology, vol. 5(9): 1276 - 82(2019)

55. Shaverdian N, Lisberg AE, Bornazyan K, Veruttipong D, Goldman JW, Formenti SC, Garon EB, Lee P. The Lancet. Oncology vol. 18(7):895903(2017)

56. Sprung CN, Forrester HB, Siva S, Martin OA. Cancer letters vol. 368(2):191-7(2015)

57. Büttner C, Skupin A, Reimann T, Rieber EP, Unteregger G, Geyer P, Frank KH. American journal of respiratory cell molecular biology vol. 17(3):315325(1997)

58. Wilson MS, Madala SK, Ramalingam TR, Gochuico BR, Rosas IO, Cheever AW, Wynn TA. The Journal of experimental medicine vol. 207(3):535-52(2010)

59. Lee CG, Homer RJ, Zhu Z, Lanone S, Wang X, Koteliansky V, Shipley JM, Gotwals P, Noble P, Chen Q, Senior RM, Elias JA. The Journal of experimental medicine vol. 194(6):809-21(2001)

60. Shibaki R, Akamatsu H, Fujimoto M, Koh Y, Yamamoto N. Annals of oncology: official journal of the European Society for Medical Oncology vol. 28(6):1404-1405(2017)

61. Manapov F, Roengvoraphoj O, Dantes M, Marschner $\mathrm{S}$, Li M, Eze C. Journal of immunotherapy (Hagerstown, Md.:1997) vol. 41(2):96-99(2018)

62. Teng $\mathrm{F}, \mathrm{Li} \mathrm{M}, \mathrm{Yu}$ J. BMC Medicine vol. 18(1):275(2020)

63. Jabbour SK, Berman AT, Decker RH, Lin Y, Feigenberg SJ, Gettinger SN, Aggarwal C, Langer CJ, Simone CB 2nd, Bradley JD, Aisner J, Malhotra J. JAMA oncology vol. 6(6):848-855(2020) 